\title{
MULTISENSOR EQUIPPED UAV/UGV FOR AUTOMATED EXPLORATION
}

\author{
S. Batzdorfer ${ }^{\text {a, }}$, M. Bobbe ${ }^{a}$, M. Becker ${ }^{a}$, H. Harms ${ }^{b}$, U. Bestmann ${ }^{a}$ \\ ${ }^{\text {a }}$ Institute of Flight Guidance, Technische Universitaet Braunschweig, Germany - \\ (s.batzdorfer, m.bobbe, m.becker, u.bestmann)@tu-braunschweig.de \\ ${ }^{\mathrm{b}}$ Institute of Mobile Machines and Commercial Vehicles, Technische Universitaet Braunschweig, Germany - \\ hannes.harms@tu-braunschweig.de
}

\begin{abstract}
KEY WORDS: UAV, UGV, GNSS/IMU positioning, 2D live image stitching, 3D reconstruction, automated operation, disaster scenarios
\end{abstract}

\begin{abstract}
:
The usage of unmanned systems for exploring disaster scenarios has become more and more important in recent times as a supporting system for action forces. These systems have to offer a well-balanced relationship between the quality of support and additional workload. Therefore within the joint research project ANKommEn - german acronym for Automated Navigation and Communication for Exploration - a system for exploration of disaster scenarios is build-up using multiple UAV und UGV controlled via a central ground station. The ground station serves as user interface for defining missions and tasks conducted by the unmanned systems, equipped with different environmental sensors like cameras - RGB as well as IR - or LiDAR. Depending on the exploration task results, in form of pictures, 2D stitched orthophoto or LiDAR point clouds will be transmitted via datalinks and displayed online at the ground station or will be processed in short-term after a mission, e.g. 3D photogrammetry. For mission planning and its execution, UAV/UGV monitoring and georeferencing of environmental sensor data, reliable positioning and attitude information is required. This is gathered using an integrated GNSS/IMU positioning system. In order to increase availability of positioning information in GNSS challenging scenarios, a GNSS-Multiconstellation based approach is used, amongst others. The present paper focuses on the overall system design including the ground station and sensor setups on the UAVs and UGVs, the underlying positioning techniques as well as 2D and 3D exploration based on a RGB camera mounted on board the UAV and its evaluation based on real world field tests.
\end{abstract}

\section{INTRODUCTION}

For an efficient planning and the realization of exploration tasks within disaster scenarios an up-to-date overview of the area of action is needed to guarantee a good situational awareness. This is especially indispensable within complex situations where a priori maps are outdated e.g. within major fire scenarios, flood areas or search-and-rescue tasks. This situational awareness is the basis for an efficient situational proceeding like e.g. path planning for fire brigades or creation of search patterns (SAR). Up to the present, this information often can only be provided by manned exploration using ground or airborne systems with the limitation of contemporary availability. The motivation of the joint research project $A N K o m m E n^{1}$ is to create an automated unmanned overall system which closes this gap with respect to providing up-to-date information of the scenario and so increases the safety of human resources by using unmanned vehicles, aerial (UAV) as well as ground based (UGV). All vehicles are equipped with identical positioning and communication hardware complemented by different environmental sensors (RGB-camera, IR camera, LiDAR) for visual exploration of the desired destination area. The visual sensor information is transmitted to a central ground station for

\footnotetext{
* Corresponding author

${ }^{1}$ The project ANKommEn (german acronym for "Automated Navigation and Communication for Exploration") is a german joint research project. Project partners are the Institute of Flight Guidance (IFF), the Institute of Mobile Machines and Commercial Vehicles (IMN) - both Technische Universitaet Braunschweig- and the AirRobot ${ }^{\circledR} \mathrm{GmbH} \&$ Co. KG, a german manufacturer of multirotor UAVs.
}

visualization and/or analyses. In order to increase the advantage using the build-up system, the unmanned systems should have a high grade of automation to reduce the workload of the operators.

Several requirements for such a system were defined in close collaboration with the associated project partners of ANKommEn, the professional fire brigade of Braunschweig and the NLWKN (Lower Saxony Water Management, Coastal Defence and Nature Conservation Agency). These institutions take a great interest in getting up-to-date information of the operational scenario and the ability to focus on a self-defined area for more detailed information. Nevertheless, the system should be easily operated without extensive training.

To meet this requirement the overall system is aspired to be highly automated and only basic inputs have to be done by the operator. For example, just by marking a destination area for exploration and choosing a predefined task and the mission will be planned automatically and the corresponding waypoint list will be sent to the vehicles, which will start the mission. Especially automated procedures of a UAV require valid position information, in particular related to accuracy, availability and continuity. To increase the availability of position information mainly in exploration areas where the UGV or the UAV operates in low altitude, whereby receiving of GNSS-Signal can be degraded by the topology (building etc.), the usage of more than one GNSS-system provides advantages. For vehicle control and georeferencing the environmental sensor data and exploration results high frequent absolute position and attitude and heading information is required. This data is gathered by fusing GNSS data and IMU measurements. 


\section{OVERALL SYSTEM DESIGN}

The overall system consists of three UAV, two UGV and a central ground and control station. The latter serves as a central human machine interface to monitor and manage cooperative operation of the UAVs/UGVs by an operator. Based on a priori known map data, exploration areas and tasks are defined and assigned to the UAVs/UGVs and will be updated with actual information of the visual sensors while performing a mission. This is done by an online 2D georeferenced photo stitching based on pictures transmitted via data link to the ground station. Path planning is then automatically conducted and transmitted via data links to the unmanned machines. The user interface developed by the IMN is capable to integrate multiple real-time sensor streams of all UAVs/UGVs to get a comprehensive overview of the scenario. Furthermore the control station can be used to adjust communication parameters like bandwidth, protocol and rate of each sensor data stream to avoid a loss of communication especially in rough terrains and to meet demands of the current mission. A scheme of the interaction and information exchange between the different vehicles and sensors is shown in Figure 1.

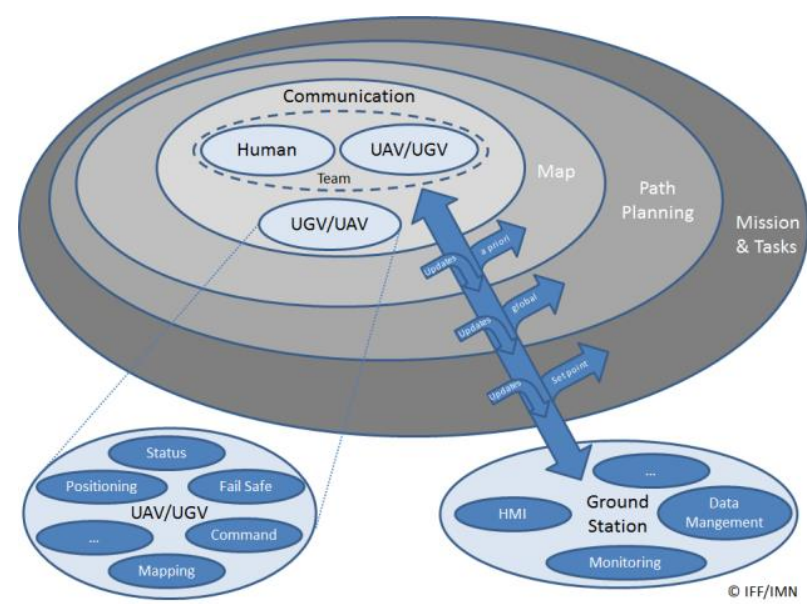

Figure 1: Diagram of interaction and information exchange

As depicted, a permanent communication link between the involved systems and the ground station is the inner basis of the overall system, for exchange of tasks, exploration data and status information of the subsystems. Definition of tasks and missions is done using the HMI of the ground station, and path planning, based on a priori known maps and predefined actions, is then conducted and transmitted to the UAV/UGV. Actual exploration results are then fed back into the ground station and can be used for further mission-planning.

For this purpose all UAVs/UGVs are equipped with a "navigation and communication unit" (NAV/COM-unit) and an "environmental sensor payload unit" (ENV-unit), including a RGB-camera, thermal camera or a LiDAR respectively.

\subsection{UAV/UGV}

The UAVs are modified hexacopter type AR200, manufactured by AirRobot ${ }^{\circledR} \mathrm{GmbH} \& \mathrm{Co}$. KG, with a payload of $2.7 \mathrm{~kg}$ (NAV/COM-unit, mounted in the upper compartment, and ENV-unit mounted under the UAV) and a corresponding flight time of up to 30 minutes depending on actual meteorological conditions (Figure 2).

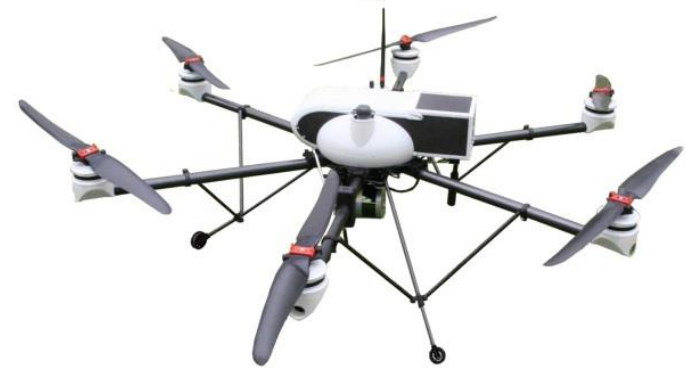

Figure 2: UAV Typ “AR200” carrying a LiDAR

The payload-sensors are carried and stabilized by a 2-axisgimbal, manufactured by AirRobot and equipped with sensors and processing units by TU Braunschweig (Figure 3).

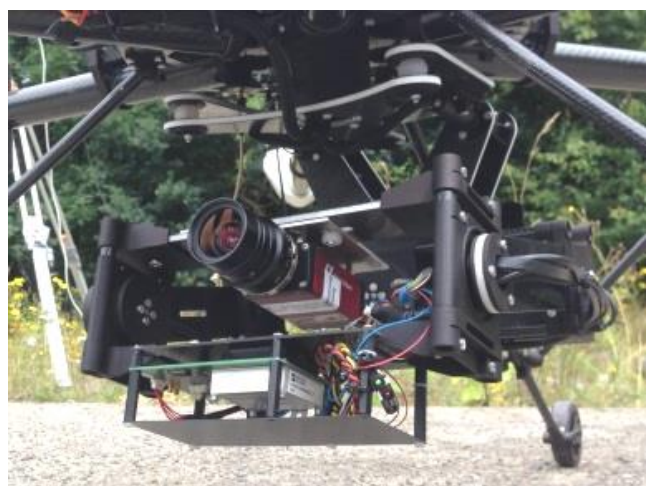

Figure 3: 2-axis-gimbal carrying a RGB-camera

For ground based exploration two Robotnik Summit XL have been chosen. (Figure 4) They have a 4-wheel-drive and are carrying a pan-tilt-zoom (PTZ) camera at the top of front chassis. Additionally the UGVs are equipped with a LiDAR and a thermal camera or stereo-RGB-camera respectively.

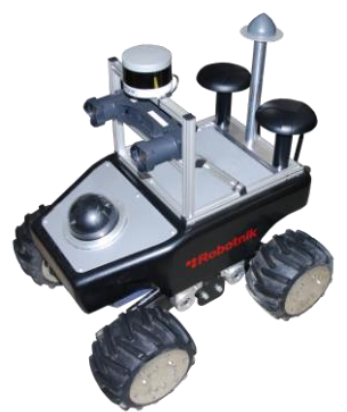

Figure 4: UGV Summit XL (Robotnik) carrying LiDAR and stereo camera

\subsection{Sensor-Hardware}

The navigation and communication unit mounted as a stack includes a network processor board type Ventana GW5520 for communication and data exchange between the UAV/UGV and the central ground and control station. For position calculation and GNSS-NTP-based Time-Server, an embedded Cortex A9 processing board type Phytec phyBOARD ${ }^{\circledR}$-Mira i.MX6 has been chosen. The data for the position calculation is provided by a custom-designed break-out-board by the Institute of Flight Guidance, which combines an Analog Devices inertial measurement unit (IMU) type ADIS16488 and a multi- 
constellation capable GNSS-Receiver type u-blox LEA-M8T (Figure 5).

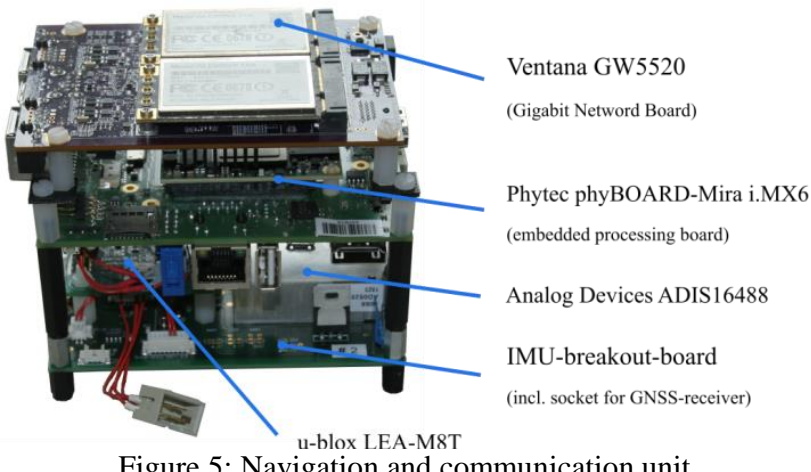

Figure 5: Navigation and communication unit

The environmental sensor payload unit is based on three different types of sensors which are interchangeable between the different UAV, using the proprietary payload interface of the AR200:

1. RBG-camera (Allied Vision Manta G-917)

2. LIDAR (Velodyne VLP-16)

3. IR-Camera (FLIR A65sc)
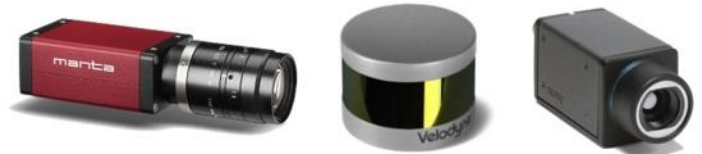

Figure 6: RGB-Camera, LiDAR and thermal camera

Concerning the UGVs, each one has a fixed environmental sensor setup. Both are equipped with a PTZ camera and a top mounted LiDAR. Additionally, one UGV carries a RGB-stereocamera, cf. Figure 4, the second one an IR camera.

Data traced by these sensors cannot be sent directly to the ground station because of the huge data amount and the limited bandwidth of the communication link. Therefore another processor-board, an Intel NUC-board, is mounted on the gimbal and connected to the visual sensor via gigabit Ethernet. The NUC-Board itself is connected to the communication board within the modified AR200. Data from the sensors are preprocessed and/or compressed on the Intel NUC and after that transmitted to the ground station

\subsection{Ground-Station}

The ground station shown in Figure 7 is the central device for command, control and visualization of the total system. It provides several options to display the data from the different sensors and vehicles (UAV/UGV) and a combination of them. Furthermore, the automated path planning for different missions and calculation of the 3D-reconstruction (photogrammetry) and online 2D stitched orthophoto is realized within it. The user can switch between various options/windows for sensor data visualization, defining missions and setting waypoints for path planning which are transmitted to desired vehicle and/or set parameters for the 3D-reconstruction, as well as monitoring the UAVs and UGVs.

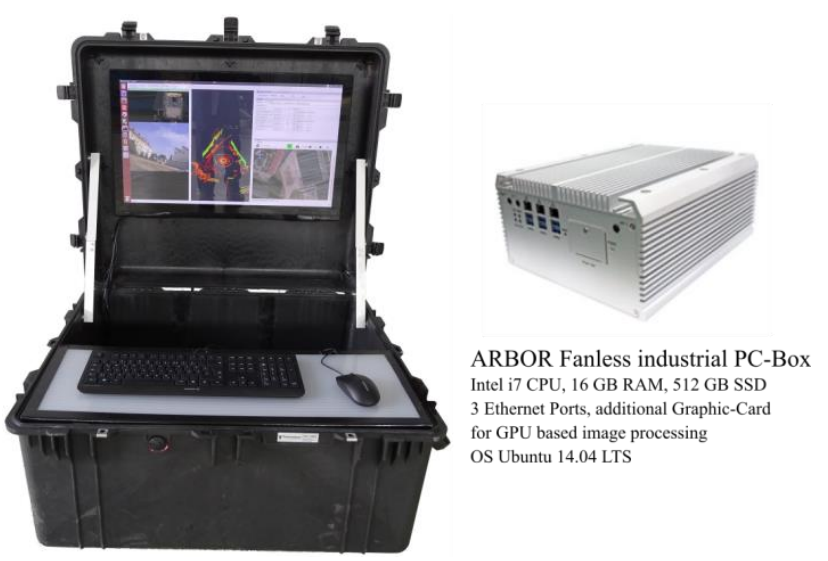

Figure 7: Ground-Station-PCBox (ARBOR FPC-7800-Series)

Figure 8 shows an example of the display while an UGV is performing a mapping mission using the top mounted LiDAR. The layout can be customized by the user.
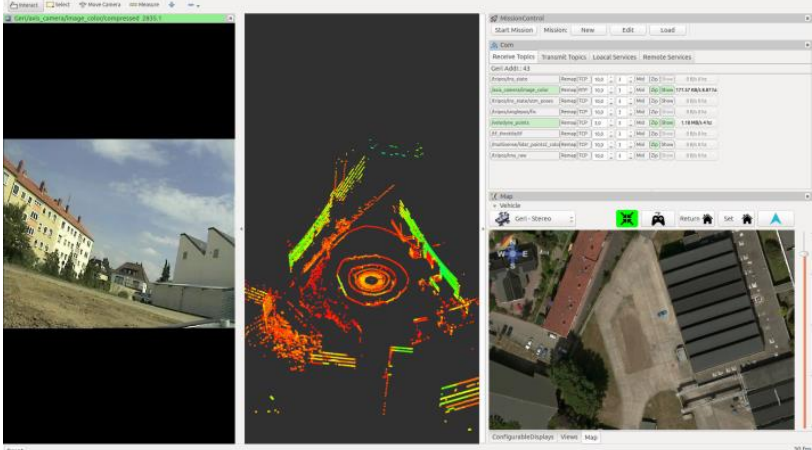

Figure 8: combined visualization of different sensors

The screenshot shows from left to right, the actual view by the PTZ camera onboard the UGV, the point cloud gathered by the LiDAR and mission parameters (top-right) as well as a map in form of an aerial view (bottom-right)

\subsection{Software-Frameworks}

The basic software for determining the vehicle's state in manner of $3 \mathrm{D}$ position, velocity, attitude and heading is established within the modular build-up navigation software framework of the Institute of Flight Guidance, with the option to process data of different sensors in real-time as well as Postprocessing for data evaluation and development purpose. Several algorithms for sensor data fusion are implemented. The algorithm which has been chosen for IMU/GNSS-Fusion is based on an Extended Kalman Filter and also provides an IMU-data-based state vector, stabilized by GNSS-Information, for the visual sensors. This state vector is published by using a ROSFramework (Robot Operating System). ROS, basically designed for applications for Robots, is a framework for inter process communication and is based on a TCP or UDP publisher/subscriber concept. The visual sensors and embedded PCs subscribe to different ROS-messages, e.g. the state-vectormessage or information of other sensors.

\section{POSITIONING OF UAV AND UGV}

Automated operation of UGV and UAVs requires valid position as well as attitude and heading information. In the case of using only one GNSS-system, e.g. GPS, the signal quality and availability can be degraded by environment (buildings) and can 
result in a less precise or even a lack of position information. In addition attitude and heading information cannot be determined when using only GNSS based positioning.

\subsection{GNSS-Multi-Constellation}

In order to overcome the risk of poor availability of GNSS based position information - for example when operating the $\mathrm{UAV} / \mathrm{UGV}$ in GNSS challenging areas - one way is parallel usage of different GNSS-systems to raise the number of received satellite signals. Today there are four satellite positioning systems available, the common GPS (actually 31 satellites (NAVCEN, 2017)) and GLONASS (actually 24 satellites (IAC, 2017)) and the evolving Galileo (actually 12 satellites (GSA, 2017)) and BeiDou (actually 14 satellites (IGS, 2017)). In respect of the fact that one is able to receive signals only of a subset of all available satellites, the usage of different constellations can increase the availability significantly. In the worst case, a multi-constellation based position is still equal to the single-constellation solution of positioning.

When using a multi-constellation approach for positioning, one has to take care of several aspects that differ between those GNSS systems (NAVCEN, 2016), (ISTC, 2008), (GSA, 2016), (BDS, 2016). All satellite based navigation systems use different geodetic reference frames and time basis. That means one has to transform measurements gathered from another GNSS-System into the reference frame of the desired system $\left(\right.$ see $\left.\mathrm{eq}^{\circ}(1)\right)$

- GPS: World Geodetic System 1984 (WGS84) (NAVCEN, 2016)

- GLONASS: Parametry Zemli 1990 (Parameter of the Earth 1990 PZ-90) (ISTC, 2008)

- Galileo: Galileo Reference Frame (GTRF) (GSA, 2016)

- BeiDou: China Geodetic Coordinate System 2000 (CGCS2000) (BDS, 2016)

All these reference frames are in compliance with the International Terrestrial Reference Frame (ITRF) and can be transferred into each other, similar to transformation of PZ90 in WGS84 in eq. (1).

$$
\left(\begin{array}{l}
X \\
Y \\
Z
\end{array}\right)_{W G S 84}=\left(\begin{array}{l}
X \\
Y \\
Z
\end{array}\right)_{P Z 90}+\left(\begin{array}{l}
-0,36 m \\
+0,08 m \\
+0,18 m
\end{array}\right)
$$

Due to the fact, that the different GNSS-systems have a different time base, a different approach is needed in order to get reliable position solutions. This inter-system offset has to be taken into account for combined position solution. A way to handle this problem is to extend the estimated state vector by adding a clock error for each GNSS-System, which leads to

$$
\vec{x}=\left(\begin{array}{c}
x \\
y \\
z \\
c \cdot \Delta t_{G P S} \\
c \cdot \Delta t_{G L O} \\
c \cdot \Delta t_{G A L} \\
c \cdot \Delta t_{B D S}
\end{array}\right)
$$

where $\quad \vec{x}=$ state vector

$x, y, z=$ position

$\Delta t_{G P S}, \Delta t_{G L O}, \Delta t_{G A L}, \Delta t_{B D S}=$ constellation specific clock error

$c=$ speed of light
By adding the different clock errors the number of unknown variables increases, so for solving this equation more GNSS measurements are required. In the case displayed in eq. (2) seven variables have to be determined. If no measurements of a specific GNSS-system are available, the estimation can be simplified by excluding the corresponding clock error from estimation.

The geometric distribution of the satellites is improved by using more than one GNSS constellation, indicated by a lower value of Dilution of Precision (DOP). On the one hand this indicates a better mapping of per-range accuracy to position accuracy which provides not necessarily a higher accuracy compared to a single constellation solution but on the other hand it will improve the overall availability and integrity.

The navigation software framework of the IFF is designed for real-time computation and also for Postprocessing. In Postprocessing the recorded real-world sensor data is streamed into the software framework with the option that one can change several parameters and settings for calculation. One option is to change the cut-off elevation for the satellites to exclude satellites at low elevation from position calculation. This parameter will be changed for simulating environmental conditions that block receiving GNSS-signals, like buildings within urban scenarios, to compare the availability of received GNSS-signals for single- and multi-constellation based position calculation. Recorded data of a real world field serve as the database for the Postprocessing with the different cut-off elevation parameters. At the beginning of the field test, there was a short time initialization period in order to boot the OS and to start basic processes for positioning. After that a predefined mission was flown. The stored GNSS measurements on board the UAV were transmitted to the ground station after the mission had been completed for described Postprocessing.

The Postprocessing has been performed with different parameters regarding the cut-off elevation for the satellites. Starting point for the comparison is cut-off elevation of $5^{\circ}$. This is a common value for GNSS-Position calculation and excludes low satellites, the signals of which on the one hand have to path a long distance through the troposphere and on the other hand are vulnerable to multipath effects for example on flat roofs.

The result is shown in Figure 9 and illustrates that the number of received GNSS-Satellites is higher than the number that are used for position calculation. This is caused by the predefined Cut-off-elevation and further quality checks like signal-tonoise-ratio aiming to eliminate the influence of model-based output of the GNSS-receiver.

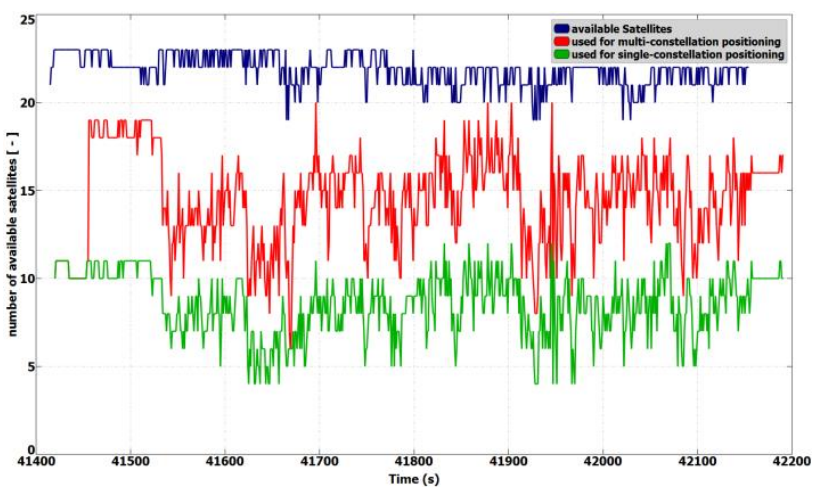

Figure 9: Number of available Satellites vs. used for position solution with cut-off elevation of $5^{\circ}$ 
At the beginning of the initialization period the number of received satellites is 23 , where 10 to 11 are used for a GPSbased single-constellation positioning, and after approximately 30 seconds when all ephemeris have been received a multiconstellation based positioning is available and the number of satellites used for positioning increases to 18-19. The number of available satellites varies during the flight of the UAV, which is caused by manoeuvres of the UAV to fulfil the predefined mission as well as compensating gusts. Overall the number of satellites used for multi-constellation based positioning is nearly twice the number of satellite of a single-constellation based positioning.

To simulate a degraded GNSS reception during the flight, the cut-off elevation is increased to $20^{\circ}$. This is comparable to e.g. a flight in an urban scenario or other areas with obstacles like trees, and it reduces the number of satellites used for a singleconstellation position solution to 7 or less and to 13 or less respectively for a multi-constellation solution (Figure 10). Compared to the deviation with a cut-of elevation of $5^{\circ}$ (Figure 9), the number varies not so much, which means that the effect of degraded GNSS reception by self-shadowing of the UAV during manoeuvres for satellites with a low elevation is minimized.

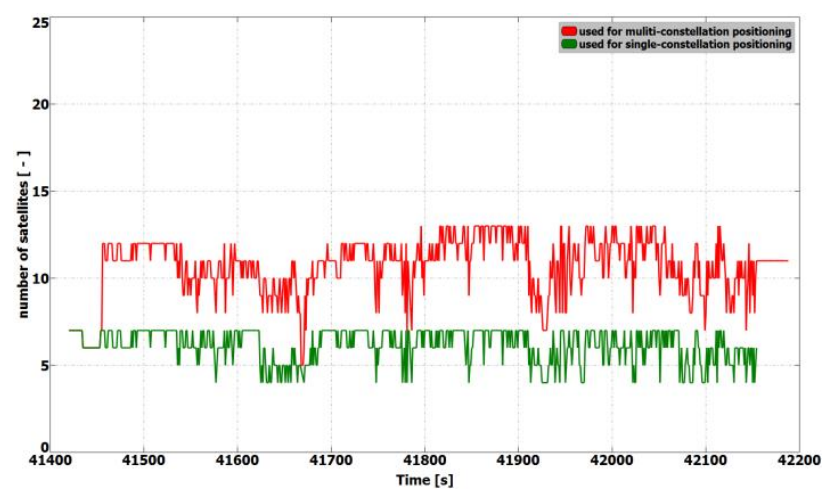

Figure 10: Number of available Satellites vs. used for position solution with cut-off elevation of $20^{\circ}$

The last case of the comparison is that only satellites with an elevation higher than $35^{\circ}$ are used for position calculation. This case is especially reasonable for an UAV operating in similar scenarios as described in the previous paragraph but working at a lower altitude so the GNSS signals might be blocked by the environment. On the other hand this can be a case for an UGV operating between buildings or other obstacles. The graphs in Figure 11 indicates that in this case the reception of GNSS signals is extremely reduced and a valid GPS based positioning starts approximate 60 seconds later than a valid positioning using a multi-constellation approach. While using only a GPS based single-constellation the number of satellites is reduced to the minimum of required satellites for position calculation of four in contrast to 5-8 available satellites for a multiconstellation based solution.

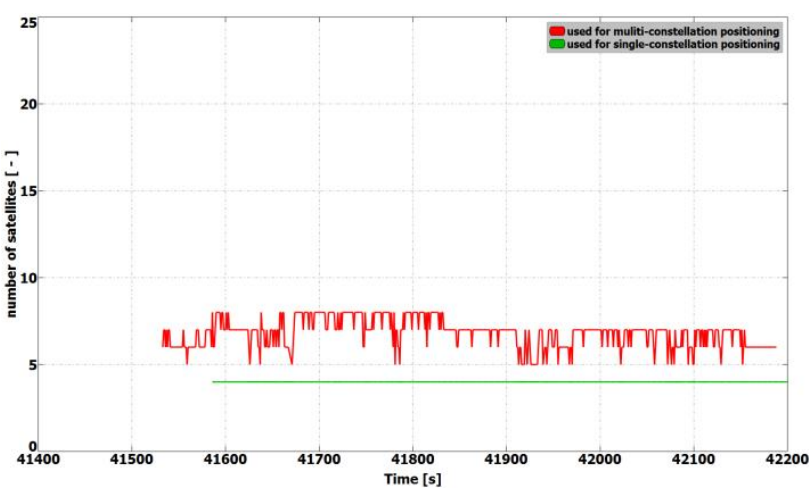

Figure 11: Number of available Satellites vs. used for position solution with of cut-off elevation of $35^{\circ}$

Especially this case shows the benefit of a higher availability of GNSS satellites by using more than one GNSS system for positioning.

\subsection{GNSS/IMU Fusion}

Using the described GNSS multi-constellation approach availability of position information could be increased. For attitude and heading determination, an Inertial Measurement Unit is nevertheless indispensable. Additionally, the frequency of the pure GNSS based positioning information usually is between $1 \mathrm{~Hz}$ to $5 \mathrm{~Hz}$ within the described hardware setup. For a meaningful georeferencing of the above described and used environmental sensors much higher frequent position and attitude information is required.

So the GNSS based positioning is used for aiding the IMUmeasurements within an extended Kalman Filter using the navigation software framework of the IFF. The mentioned ADIS IMU provides high frequent, three dimensional measurements of accelerations and angular rates. Using common strapdown algorithm processing, high frequent position, velocity, attitude and heading information is provided in real time. Due to the short time stability of pure inertial navigation, the GNSS positioning results are used for aiding purpose within the Kalman Filter's update step. To overcome the absence of GNSS aiding information even when using multi-constellation, mainly two options are possible. Firstly, a short coasting period is possible after the data fusion has reached steady state. Secondly, it is possible due to the high modularly design of the IFF's navigation software framework to use position and/or attitude increments from environmental sensor data processing for aiding the IMU.

The determined vehicle's state vector is then distributed with high frequency within the system for georeferencing measurements of the used environmental sensors especially the RGB camera and the LiDAR for photogrammetry and SLAM applications.

\section{PHOTOGRAMMETRY AND SLAM}

When thinking of major fire scenarios, up-to-date information is required, whereas a priori information like maps could be outof-date. Therefore techniques have been developed to gather a 2D overview based on single RGB pictures taken and processed on-board an UAV and transmitted to the ground station via data links. Additional processing of a 3D-reconstruction of the scenario is an integrated feature within the ground station. Both approaches were implemented with the goal to get an automated rapid aerial mapping solution. 
In the case of the 2D overview, SLAM algorithms, often used in robotic research, are adapted for this specific use-case. These algorithms provide good results for a rapid aerial mapping solution to get an overview of the scenario, because the map is updated incrementally with every new image, but they are less precise, which can be compensated by using the photogrammetric 3D-reconstruction. The live mapping (SLAM) approach is based on the ORB-SLAM algorithm and the photogrammetry based approach uses the commercially available photogrammetry software Agisoft Photoscan.

The systems, on the UAV for $2 \mathrm{D}$ and for $3 \mathrm{D}$ on the ground station, use the ROS-Framework for processing the visual sensor data and the described techniques for positioning, georeferencing and attitude determination. For data exchange between these Frameworks several software interfaces have been implemented. Figure 12 displays a flowchart of the implemented workflow.

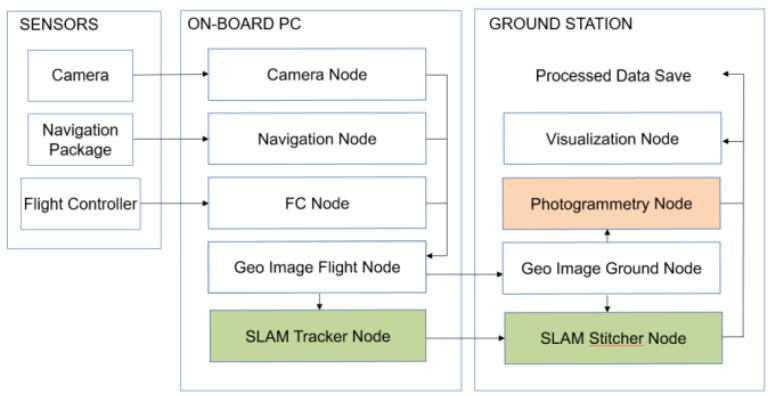

Figure 12: ROS node layout with SLAM (green) and Photogrammetry workflow (red)

The sensor/input data is received by corresponding nodes on the aerial vehicle. After adding the camera pose information to the image in the Geo Image Flight Node, the image is sent to the Geo Image Ground Node on the Ground Station. The SLAM process is separated into two parts. The SLAM Tracker Node calculates the transformation between images and the SLAM Stitcher Node applies the transformations. The transformed images are displayed by the Visualization Node. The Photogrammetry Node receives the georeferenced images, stores the data and initiates the photogrammetric processing ones the survey is finished. The results can also be displayed by the Visualization Node and exported in a desired format.

\subsection{Visual SLAM}

During the past few years computer vision, especially SLAM (Simultaneous localization and mapping) based algorithms have developed rapidly. In 2007 Klein and Murray presented a method to estimate a pose by using monocular image processing, known as Parallel Tracking and Mapping (PTAM). On the one hand, they integrated a bundle adjustment $(B A)$ and on the other hand separated the tracking and the mapping procedure into different threads which led to a real-time capable framework (Klein, 2007). Mur-Artal and Montiel used these basic principles of PTAM and integrated a robust loop closing and another method of relocalization, which is known as ORB SLAM 2 (Oriented FAST and Rotated BRIEF). The structure of the ORB SLAM is shown in Figure 13.

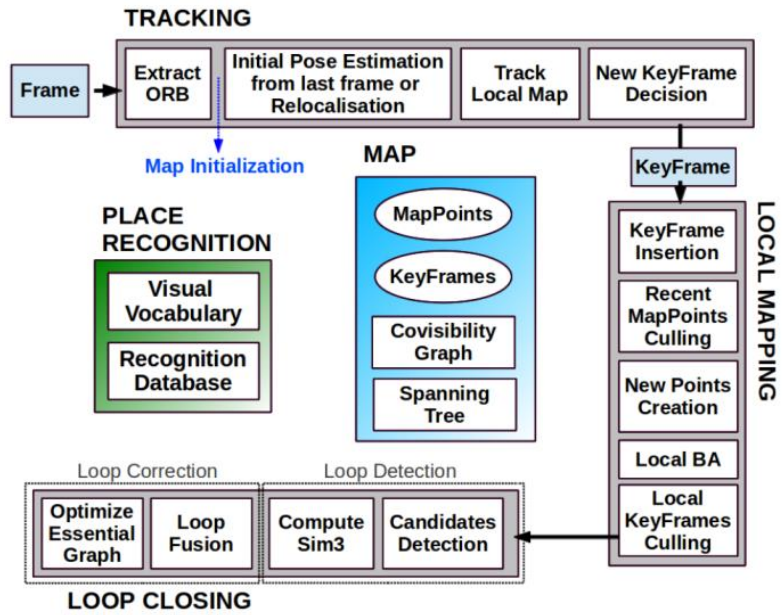

Figure 13: ORB SLAM system overview (Mur-Artal, 2015)

Figure 13 shows the approach of separating the Tracking, Local Mapping and the Loop Closing into different threads (gray boxes) and the main map and place recognition in the middle. The tracking thread predicts the current pose from the last known position and movement by using a constant velocity model and performs a guided search of map points. If these points are found near the estimated position, the velocity model is valid and the tracking procedure continues. Otherwise the tracking is lost and a relocalization in the global map starts by using a subset of features, which are increased after detection of corresponding features in other keyframes to optimize the camera pose and finally, the tracking procedure continues. The last step of this procedure is to decide whether the current frame contains enough information to be inserted as a new keyframe for further calculations. To mark a frame as a new keyframe, the frame must fulfill all of the following conditions:

1) More than minimum number of frames has passed

2) Local mapping is on idle or condition 1 fulfilled

3) A minimum number of 50 points is observed

4) A maximum of $90 \%$ of the features is already observed by the other frames.

When a new keyframe is passed to the local mapping procedure and is inserted as node into a covisibility graph structure, new correspondences are searched in the connected keyframes to triangulate new points. Based on the information accumulated during the tracking, a point culling is done in order to keep only high quality points in the map as well as a culling of redundant keyframes.

After finishing the keyframe culling in the local mapping process, a loop closing is performed. This is one of the main improvements compared to PTAM. If a loop is detected the drift accumulated in the loop is computed, and both sides of the loop are aligned and visible points are fused. In a final step a pose graph optimization is done to achieve global consistency.

This information of the 3D camera pose is used to generate a 2D orthophoto in real-time while the vehicle is flying. To create a 2D orthophoto, a common reference frame is approximated, which is orthogonal to all camera measurements. The projection is performed by using a projection model based on a pinhole camera. 


$$
\underline{x}=\underline{R^{1}}(\underline{\underline{K}} s \underline{u t})
$$

where

$$
\begin{aligned}
& \frac{x}{R^{1}}=\text { world point } \\
& \frac{\bar{K}}{\underline{\bar{K}}}=\text { exterior orientation }(3 \times 3) \\
& \underline{\bar{u}}=\text { point in homographic coordinates (image plain) } \\
& \underline{\underline{s}}=\text { exterior position of the camera } \\
& =\text { scale factor of projection direction vector }
\end{aligned}
$$

For a compensation of geometric distortion caused by the lens, image point can be distorted by using

$$
\begin{aligned}
& x_{\text {cor }}=x+\left[2 p_{1} x y+p_{2}\left(r^{2}+2 x^{2}\right)\right] \\
& y_{\text {cor }}=y+\left[p_{1}\left(r^{2}+2 y^{2}\right)+2 p_{1} x y\right]
\end{aligned}
$$

After the compensation and distortion the whole images can be stitched to the current global map.

\subsection{Photogrammetry}

This approach uses off-the-shelf photogrammetric processing software. The processing is triggered automatically when the survey is completed and all images are transferred over the Wi-Fi network. To ensure an acceptable compromise between orthophoto quality and the required processing time, an analysis regarding the impact of the most relevant processing parameters has been performed, cf. section 4.2.1. The workflow is implemented using the Agisoft Photoscan API. For georeferencing of the images, the camera location and the inner camera geometry were written to the EXIF file of each image by the Geo Image Ground Node (Figure 12).

The process of the Photogrammetry consists of 4 steps:

1. Camera alignment (optimize the homographic equation)

2. Mesh by generated tiepoint

3. Orthophoto (dense cloud or Digital Elevation Model)

4. Export

\subsubsection{Performed Profile Analyses and Evaluation}

To determine quality parameters four profiles were defined and are given in Table 1.

\begin{tabular}{|l|c|c|}
\hline profile & alignment accuracy & mesh face count \\
\hline \hline Agi lowest & lowest & lowest \\
\hline Agi low & low & low \\
\hline Agi medium & medium & medium \\
\hline Agi high & high & high \\
\hline
\end{tabular}

Table 1: Photogrammetry parameter profile definition

To validate and compare the created results, the system was tested by flying a sample mission. The accuracy of the created maps is compared by using three Ground Reference Points (GRPs) which were aligned to the corners of a building (Figure 15). An overlap of $80 \%$ and a sidelap of $70 \%$ were chosen to guarantee a robust processing. To identify the GRP, a Ground Sampling Distance (GSD) of $2 \mathrm{~cm}$ was desired. This resulted in a mission consisting of 4 times $400 \mathrm{~m}$ lines with a distance of $40 \mathrm{~m}$ and an altitude of $70 \mathrm{~m}$ over ground. During the flight (time of flight approx. 12 minutes) 160 images were created. To compare the presented profiles, they were triggered one after another. All defined profiles resulted in consistent solutions and were successfully georeferenced. (Figure 14)

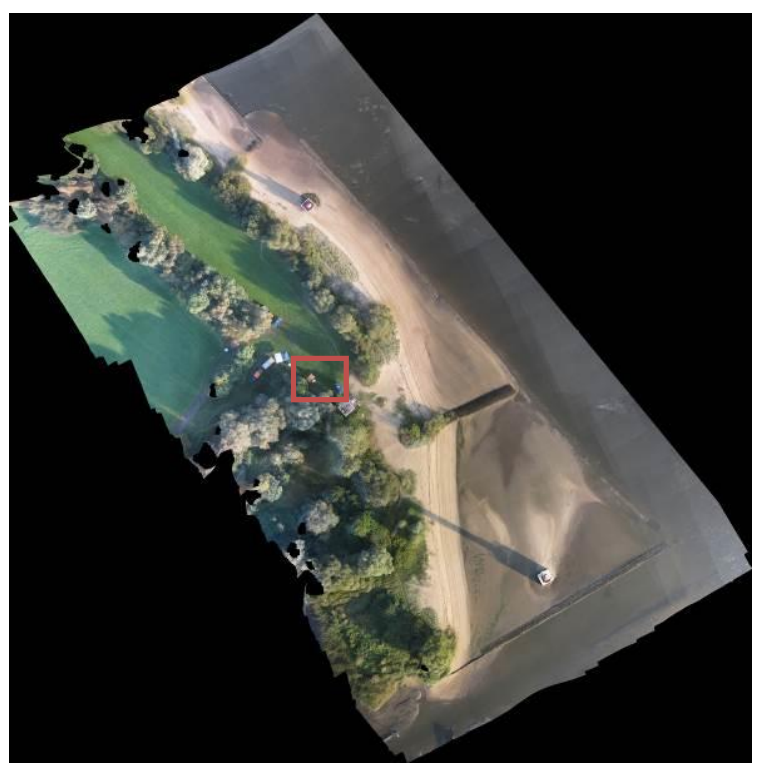

Figure 14: Orthophoto created with the AgiSoft (high profil)

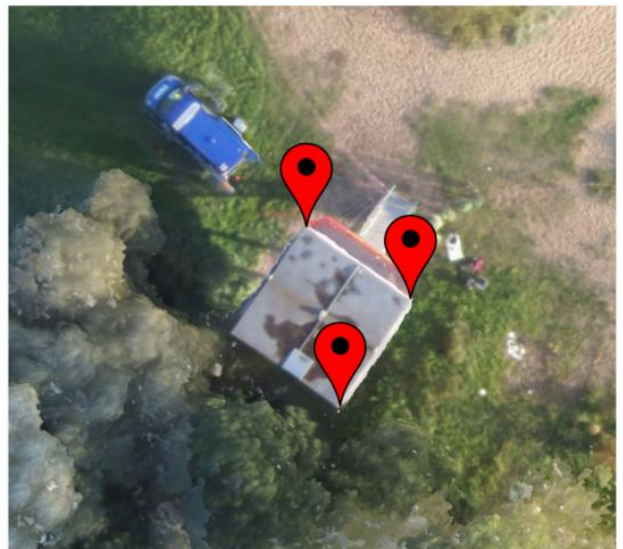

Figure 15: Reference-Points (red marked area of Figure 14)

The map based on the lowest profile could not recreate the complete area. The remaining profiles led to similar results without notable differences to visual inspection. The processing time varied between 9 (lowest), 14 (low), 132 (medium) and 700 (high) minutes. The horizontal mean error to the reference measurement is given in Figure 16.

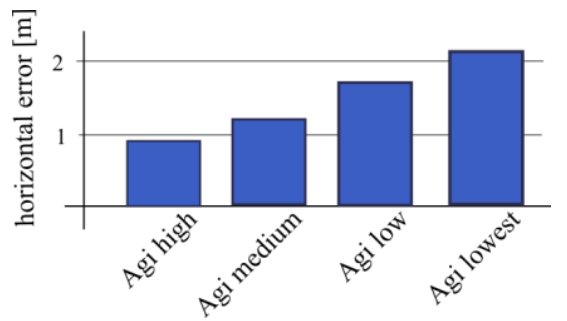

Figure 16: Horizontal error to GRPs of the different profiles

The mean error in the Agi high profile with a value of $1 \mathrm{~m}$ is half as high as in the lowest profile with a mean error of $2.1 \mathrm{~m}$. The calculated errors using the Agi medium and Agi low profiles are in between with an error of $1.2 \mathrm{~m}$ for the medium and $1.7 \mathrm{~m}$ for the low profile.

To evaluate the correct workflow of both approaches of 2Dlive-stitching and the 3D-photogrammetry, a real world flight test above an agricultural crop land has been performed and the 
results of both approaches are shown in Figure 17 and Figure 18. Generally, agricultural crop land and its mean textured surface pose a challenge for mapping processes because of the limited number of trackable features.

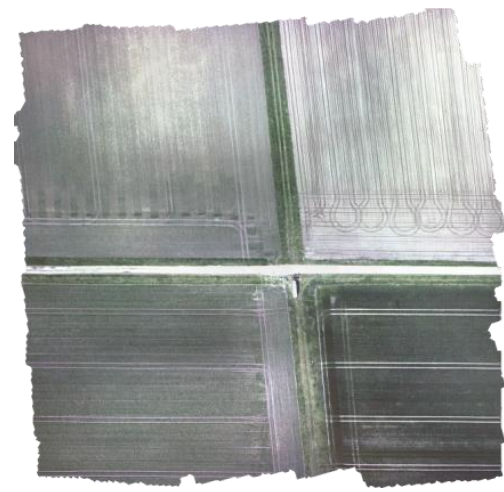

Figure 17: photogrammetry result

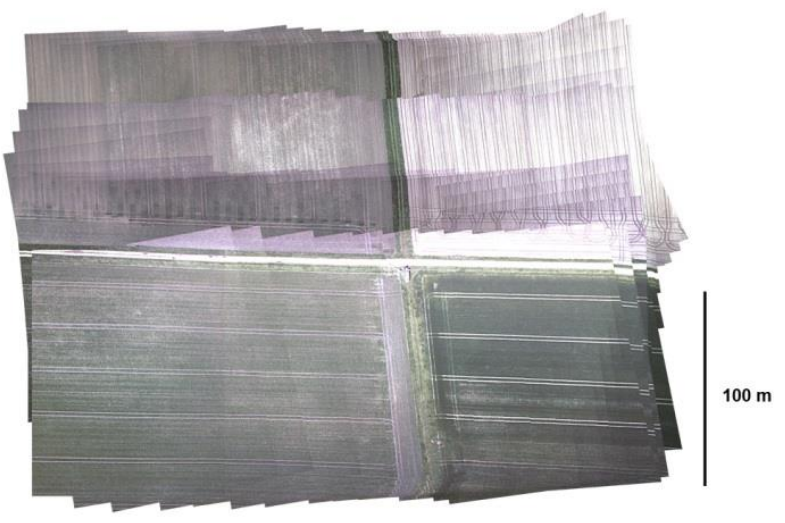

Figure 18: result of the SLAM approach

Both implemented approaches were successfully integrated to get the desired full automated rapid aerial mapping solution. This also includes the basic tasks of the automated mission planning, camera control, image transport to ground station, automated processing and the visualization of the results.

The 3D-photogrammetry provides a more detailed image (Figure 17) compared to the image of the 2D-live-stichting approach (Figure 18), but both approaches are capable to provide the desired information of the area.

\section{CONCLUSION}

In this paper, the benefits of a multi-constellation GNSS based positioning have been demonstrated with a focus on UAVs and UGVs operating in catastrophic scenarios, especially in situations where a GNSS signal reception might be blocked by obstacles or the environment. This position information is also used for georeferencing of images and therefore for a visual reconstruction of the exploration area. The introduced overall system has demonstrated the capability of an automated orthophoto generation. Both implemented mapping methods, a 2D-live-stitching and a 3D-photogrammetry, provided results which fulfil the requirements to get an instantaneous 2Doverview and a contemporary $3 \mathrm{D}$-reconstruction of the area.

\section{ACKNOWLEDGEMENTS}

The presented work was done within the joint research project "ANKommEn" funded by the German Federal Ministry of Economic Affairs and Energy administrated by the Space Administration of the DLR (funding code: 50NA1518).

\begin{tabular}{l|l} 
Federal Ministry \\
for Economic Affairs \\
and Energy
\end{tabular}

\section{REFERENCES}

BDS, 2016. BeiDou Navigation Satellite System , Signal in Space, Interface Control Document, Open Service Signal (Version 2.1), November 2016

GSA, 2016. European GNSS (Galileo) Open Service: Signal-inSpace Interface Control Document, OS SIS ICD V1.3, December 2016

GSA, 2017. European GNSS Service Center. Constellation Information. 08.07.2017.

URL:http://www.gsc-europa.eu/system-status/ConstellationInformation

IAC, 2017. Information-Analytical Centre, Federal Space Agency. GLONASS constellation status. 08.07.2017. URL: https://www.glonass-iac.ru/en/GLONASS/

IGS, 2017. International GNSS service. MGEX - BeiDou Satellites. 08.07.2017.

URL: http://mgex.igs.org/IGS_MGEX_Status_BDS.html

ISTC, 2008. Russian Institute of Space Device Engineering, „Global Navigation Satellite System GLONASS: Interface Control Document. Edition 5.1,“ 2008

Klein, G., Murray, D., 2007, "Parallel tracking and mapping for small AR workspaces," in IEEE and ACM International Symposium on Mixed and Augmented Reality (ISMAR), Nara, Japan, November 2007, pp. 225-234.

Mur-Artal, R., Montiel, J. M. M., Tardós, J. D., 2015, "ORBSLAM: A Versatile and Accurate Monocular SLAM System," in IEEE Transactions on Robotics, vol. 31, no. 5, October 2015, pp. 1147-1163

NAVCEN, 2016. Global Positioning Systems Directorate Systems Engineering \& Integration, Interface Specification, IRN-IS-200H-003, 28 July 2016

NAVCEN, 2017. U.S. Department of Homeland Security. GPS constellation status. 08.07.2017.

URL: http://www.navcen.uscg.gov/?Do=constellationStatus 